\title{
Denis Diderot
}

\section{"Autorité politique"}

\author{
Article de l'Encyclopédie
}

\section{$(1751-1765)$}

Un document produit en version numérique par Denis Collin, bénévole, docteur ès lettres et sciences humaines,

Professeur agrégé de philosophie, au Lycée Aristide Briand à Evreux (Eure),

Courriel : denis.collin@wanadoo.fr

Site web : http://perso.wanadoo.fr/denis.collin

Dans le cadre de la collection: "Les classiques des sciences sociales" dirigée et fondée par Jean-Marie Tremblay, professeur de sociologie au Cégep de Chicoutimi

Site web: http://www.uqac.uquebec.ca/zone30/Classiques_des_sciences_sociales/index.html

Une collection développée en collaboration avec la Bibliothèque Paul-Émile-Boulet de l'Université du Québec à Chicoutimi Site web: http://bibliotheque.uqac.uquebec.ca/index.htm 
Un document produit en version numérique par Denis Collin, bénévole, docteur ès lettres et sciences humaines,

Professeur agrégé de philosophie, au Lycée Aristide Briand à Évreux (Eure),

Courriel : denis.collin@wanadoo.fr

Site web : http://perso.wanadoo.fr/denis.collin

à partir de :

\section{Denis Diderot}

"Autorité politique"

Article de 1'Encyclopédie

Une édition électronique réalisée à partir de l'article de l'Encyclopédie, "Droit naturel " in Oeuvres en 5 volumes édité chez Robert Laffont - collection Bouquins par Laurent Versini. Volume III.

Polices de caractères utilisée :

Pour le texte: Times, 12 points.

Pour les citations : Times 10 points.

Pour les notes de bas de page : Times, 10 points.

Édition électronique réalisée avec le traitement de textes Microsoft Word 2000 .

Mise en page sur papier format

LETTRE (US letter), 8.5' x 11'")

Édition complétée le 20 janvier 2003 à Chicoutimi, Québec.

\section{E Fait avec}

Macintosh 


\title{
Article "Autorité politique"
}

\section{(Encyclopédie).}

\begin{abstract}
AUTORITÉ POLITIQUE. Aucun homme n'a reçu de la nature le droit de commander aux autres. La liberté est un présent du ciel, et chaque individu de la même espèce a le droit d'en jouir aussitôt qu'il jouit de la raison. Si la nature a établi quelque autorité, c'est la puissance paternelle : mais la puissance paternelle a ses bornes, et dans l'état de nature elle finirait aussitôt que les enfants seraient en état de se conduire. Toute autre autorité vient d'une autre origine que de la nature. Qu'on examine bien, et on la fera toujours remonter à l'une de ces deux sources : ou la force et la violence de celui qui s'en est emparé, ou le consentement de ceux qui s'y sont soumis par un contrat fait ou supposé entre eux et celui à qui ils ont déféré l'autorité.
\end{abstract}

La puissance qui s'acquiert par la violence n'est qu'une usurpation, et ne dure qu'autant que la force de celui qui commande l'emporte sur celle de ceux qui obéissent ; en sorte que si ces derniers deviennent à leur tour les plus forts et qu'ils secouent le joug, ils le font avec autant de droit et de justice que l'autre qui le leur avait imposé. La même loi qui a fait l'autorité, la défait alors : c'est la loi du plus fort. 
Quelquefois l'autorité qui s'établit par la violence change de nature c'est lorsqu'elle continue et se maintient du consentement exprès de ceux qu'on a soumis ; mais elle rentre par là dans la seconde espèce dont je vais parler ; et celui qui se l'était arrogée, devenant alors prince, cesse d'être tyran.

La puissance qui vient du consentement des peuples suppose nécessairement des conditions qui en rendent l'usage légitime, utile à la société, avantageux à la république, et qui la fixent et la restreignent entre des limites : car l'homme ne doit ni ne peut se donner entièrement et sans réserve à un autre homme, parce qu'il a un maître supérieur au-dessus de tout, à qui seul il appartient tout entier. C'est Dieu, dont le pouvoir est toujours immédiat sur la créature, maître aussi jaloux qu'absolu, qui ne perd jamais de ses droits, et ne les communique point. Il permet pour le bien commun et pour le maintien de la société, que les hommes établissent entre eux un ordre de subordination, qu'ils obéissent à l'un d'eux : mais il veut que ce soit par raison et avec mesure, et non pas aveuglément et sans réserve, afin que la créature ne s'arroge pas les droits du Créateur. Toute autre soumission est le véritable crime d'idolâtrie. Fléchir le genou devant un homme ou devant une image n'est qu'une cérémonie extérieure, dont le vrai Dieu qui demande le cœur et l'esprit ne se soucie guère, et qu'il abandonne à l'institution des hommes pour en faire, comme il leur conviendra, des marques d'un culte civil et politique, ou d'un culte de religion. Ainsi ce ne sont point ces cérémonies en elles-mêmes, mais l'esprit de leur établissement qui en rend la pratique innocente ou criminelle. Un Anglais n'a point de scrupule à servir le roi le genou en terre ; le cérémonial ne signifie que ce qu'on a voulu qu'il signifiât ; mais livrer son cœur, son esprit et sa conduite sans aucune réserve à la volonté et au caprice d'une pure créature, en faire l'unique et le der-nier motif de ses actions, c'est assurément un crime de lèse-majesté divine au premier chef : autrement ce pouvoir de Dieu, dont on parle tant, ne serait qu'un vain bruit dont la politique humaine userait à sa fantaisie, et dont l'esprit d'irréligion pourrait se jouer à son tour ; de sorte que toutes les idées de puissance et de subordination venant à se confondre, le prince se jouerait de Dieu, et le sujet du prince.

La vraie et légitime puissance a donc nécessairement des bornes. Aussi l'Écriture nous dit-elle: "Que votre soumission soit raisonnable», sit rationabile obsequium vestrum. «Toute puissance qui vient de Dieu est une puissance réglée », omnis potestas a Deo ordinata est. Car c'est ainsi qu'il faut entendre ces paroles, conformément à la droite raison et au sens littéral, et non conformément à l'interprétation de la bassesse et de la flatterie, qui prétendent que toute puissance, quelle qu'elle soit, vient de Dieu. Quoi donc, n'y a-t-il point de puissances injustes ? n'y a-t-il pas des autorités qui, loin de 
venir de Dieu, s'établissent contre ses ordres et contre sa volonté ? les usurpateurs ont-ils Dieu pour eux ? faut-il obéir en tout aux persécuteurs de la vraie religion ? et pour fermer la bouche à l'imbécillité, la puissance de l'Antéchrist sera-t-elle légitime ? Ce sera pourtant une grande puissance. Énoch et Élie qui lui résisteront, seront-ils des rebelles et des séditieux qui auront oublié que toute puissance vient de Dieu, ou des hommes raisonnables, fermes et pieux, qui sauront que toute puissance cesse de l'être dès qu'elle sort des bornes que la raison lui a prescrites, et qu'elle s'écarte des règles que le souverain des princes et des sujets a établies ; des hommes enfin qui penseront; comme saint Paul, que toute puissance n'est de Dieu qu'autant qu' elle est juste et réglée ?

Le prince tient de ses sujets mêmes l'autorité qu'il a sur eux ; et cette autorité est bornée par les lois de la nature et de l'État. Les lois de la nature et de l'État sont les conditions sous lesquelles ils se sont soumis, ou sont censés s'être soumis à son gouvernement. L'une de ces conditions est que n'ayant de pouvoir et d'autorité sur eux que par leur choix et de leur consentement, il ne peut jamais employer cette autorité pour casser l'acte ou le contrat par lequel elle lui a été déférée : il agirait dès lors contre lui-même, puisque son autorité ne peut subsister que par le titre qui l'a établie. Qui annule l'un détruit l'autre. Le prince ne peut donc pas disposer de son pouvoir et de ses sujets sans le consentement de la nation, et indépendamment du choix marqué dans le contrat de soumission. S'il en usait autrement, tout serait nul, et les lois le relèveraient des promesses et des serments qu'il aurait pu faire, comme un mineur qui aurait agi sans connaissance de cause, puisqu'il aurait prétendu disposer de ce qu'il n'avait qu'en dépôt et avec clause de substitution, de la même manière que s'il l'avait eu en toute propriété et sans aucune condition.

D'ailleurs le gouvernement, quoique héréditaire dans une famille, et mis entre les mains d'un seul, n'est pas un bien particulier, mais un bien public, qui par conséquent ne peut jamais être enlevé au peuple, à qui seul il appartient essentiellement et en pleine propriété. Aussi est-ce toujours lui qui en fait le bail : il intervient toujours dans le contrat qui en adjuge l'exercice. Ce n'est pas l'État qui appartient au prince, c'est le prince qui appartient à l'État ; mais il appartient au prince de gouverner dans l'État, parce que l'État l'a choisi pour cela, qu'il s'est engagé envers les peuples à l'administration des affaires, et que ceux-ci de leur côté se sont engagés à lui obéir conformément aux lois. Celui qui porte la couronne peut bien s'en décharger absolument s'il le veut; mais il ne peut la remettre sur la tête d'un autre sans le consentement de la nation qui l'a mise sur la sienne. En un mot, la couronne, le gouvernement, et l'autorité publique, sont des biens dont le corps de la nation est propriétaire, et dont les princes sont les usufruitiers, les ministres et les dépositaires. Quoique chefs de l'État, ils n'en sont pas moins membres, à la vérité les premiers, les 
plus vénérables et les plus puissants, pouvant tout pour gouverner, mais ne pouvant rien légitimement pour changer le gouvernement établi, ni pour mettre un autre chef à leur place. Le sceptre de Louis XV passe nécessairement à son fils aîné, et il n'y a aucune puissance qui puisse s'y opposer : ni celle de la nation, parce que c'est la condition du contrat, ni celle de son père par la même raison.

Le dépôt de l'autorité n'est quelquefois que pour un temps limité, comme dans la république romaine. Il est quelquefois pour la vie d'un seul homme, comme en Pologne; quelquefois pour tout le temps que subsistera une famille, comme en Angleterre ; quelquefois pour le temps que subsistera une famille par les mâles seulement, comme en France.

Ce dépôt est quelquefois confié à un certain ordre dans la société ; quelquefois à plusieurs choisis de tous les ordres, et quelquefois à un seul.

Les conditions de ce pacte sont différentes dans les différents États. Mais partout la nation est en droit de maintenir envers et contre tous le contrat qu'elle a fait ; aucune puissance ne peut le changer ; et quand il n'a plus lieu, elle rentre dans le droit et dans la pleine liberté d'en passer un nouveau avec qui et comme il lui plait. C'est ce qui arriverait en France, si par le plus grand des malheurs la famille entière régnante venait à s'éteindre jusque dans ses moindres rejetons ; alors le sceptre et la couronne retourneraient à la nation.

Il semble qu'il n'y ait que des esclaves dont l'esprit serait aussi borné que le cœur serait bas, qui pussent penser autrement. Ces sortes de gens ne sont nés ni pour la gloire du prince, ni pour l'avantage de la société : ils n'ont ni vertu, ni grandeur d'âme. La crainte et l'intérêt sont les ressorts de leur conduite. La nature ne les produit que pour servir de lustre aux hommes vertueux ; et la Providence s'en sert pour former les puissances tyranniques, dont elle châtie pour l'ordinaire les peuples et les souverains qui offensent Dieu ; ceux-ci en usurpant, ceux-là en accordant trop à l'homme de ce pouvoir suprême que le Créateur s'est réservé sur la créature.

L'observation des lois, la conservation de la liberté et l'amour de la patrie sont les sources fécondes de toutes grandes choses et de toutes belles actions. Là se trouvent le bonheur des peuples, et la véritable illustration des princes qui les gouvernent. Là l'obéissance est glorieuse, et le commandement auguste. Au contraire, la flatterie, l'intérêt particulier et l'esprit de servitude sont l'origine de tous les maux qui accablent un État, et de toutes les lâchetés qui le déshonorent. Là les sujets sont misérables, et les princes haïs ; là le monarque ne s'est jamais entendu proclamer le bien-aimé ; la soumission y est hon- 
teuse, et la domination cruelle. Si je rassemble sous un même point de vue la France et la Turquie, j'aperçois d'un côté une société d'hommes que la raison unit, que la vertu fait agir, et qu'un chef également sage et glorieux gouverne selon les lois de la justice; de l'autre, un troupeau d'animaux que l'habitude assemble, que la loi de la verge fait marcher, et qu'un maître absolu mène selon son caprice.

Mais pour donner aux principes répandus dans cet article toute l'autorité qu'ils peuvent recevoir, appuyons-les du témoignage d'un de nos plus grands rois. Le discours qu'il tint à l'ouverture de l'assemblée des notables de 1596, plein d'une sincérité que les souverains ne connaissent guère, était bien digne des sentiments qu'il y porta. «Persuadé, dit M. de Sully, que les rois ont deux souverains, Dieu et la loi, que la justice doit présider sur le trône, et que la douceur doit être assise à côté d'elle, que Dieu étant le vrai propriétaire de tous les royaumes, et les rois n'en étant que les administrateurs, ils doivent représenter aux peuples celui dont ils tiennent la place, qu'ils ne régneront comme lui, qu'autant qu'ils régneront en pères, que dans les États monarchiques héréditaires, il y a une erreur qu'on peut appeler aussi héréditaire, c'est que le souverain est maître de la vie et des biens de tous ses sujets, que moyennant ces quatre mots, tel est notre plaisir, il est dispensé de manifester les raisons de sa conduite, ou même d'en avoir ; que, quand cela serait, il n'y a point d'imprudence pareille à celle de se faire haïr de ceux auxquels on est obligé de confier à chaque instant sa vie, et que c'est tomber dans ce malheur que d'emporter tout de vive force. Ce grand homme, persuadé, dis-je, de ces principes que tout l'artifice du courtisan ne bannira jamais du cœur de ceux qui lui ressembleront, déclara que, pour éviter tout air de violence et de contrainte, il n'avait pas voulu que l'assemblée se fit par des députés nommés par le souverain, et toujours aveuglément asservis à toutes ses volontés ; mais que son intention était qu' on y admît librement toutes sortes de personnes, de quelque état et condition qu'elles pussent être, afin que les gens de savoir et de mérite eussent le moyen d'y proposer sans crainte ce qu'ils croiraient nécessaire pour le bien public ; qu'il ne prétendait encore en ce moment leur prescrire aucune borne ; qu'il leur enjoignait seulement de ne pas abuser de cette permission pour l'abaissement de l'autorité royale, qui est le principal nerf de l'État ; de rétablir l'union entre ses membres ; de soulager les peuples ; de décharger le trésor royal de quantité de dettes, auxquelles il se voyait sujet sans les avoir contractées; de modérer avec la même justice les pensions excessives, sans faire tort aux nécessaires, afin d'établir pour l'avenir un fonds suffisant et clair pour l'entretien des gens de guerre. Il ajouta qu'il n'aurait aucune peine à se soumettre à des moyens qu'il n'aurait point imaginés luimême, d'abord qu'il sentirait qu'ils avaient été dictés par un esprit d'équité et de désintéressement ; qu'on ne le verrait point chercher dans son âge, dans son 
expérience et dans ses qualités personnelles, un prétexte bien moins frivole que celui dont les princes ont coutume de se servir pour éluder les règlements ; qu'il montrerait au contraire par son exemple, qu'ils ne regardent pas moins les rois pour les faire observer, que les sujets, pour s'y soumettre. » « Si je faisais gloire, continua-t-il, de passer pour un excellent orateur, j'aurais apporté ici plus de belles paroles que de bonne volonté : mais mon ambition tend à quelque chose de plus haut que de bien parler. J'aspire au glorieux titre de libérateur et de restaurateur de la France. Je ne vous ai point ici appelés, comme faisaient mes prédécesseurs, pour vous obliger d'approuver aveuglément mes volontés : je vous ai fait assembler pour recevoir vos conseils, pour les croire, pour les suivre; en un mot, pour me mettre en tutelle entre vos mains. C'est une envie qui ne prend guère aux rois, aux barbes grises et aux victorieux comme moi ; mais l'amour que je porte à mes sujets, et l'extrême désir que j'ai de conserver mon État, me font trouver tout facile et tout honorable. »

«Ce discours achevé, Henri se leva et sortit, ne laissant que M. de Sully dans l'assemblée, pour y communiquer les états, les mémoires et les papiers dont on pouvait avoir besoin. »

On n'ose proposer cette conduite pour modèle, parce qu'il y a des occasions où les princes peuvent avoir moins de déférence, sans toutefois s'écarter des sentiments qui font que le souverain dans la société se regarde comme le père de famille, et ses sujets comme ses enfants. Le grand monarque que nous venons de citer nous fournira encore l'exemple de cette sorte de douceur mêlée de fermeté, si requise dans les occasions où la raison est si visiblement du côté du souverain qu'il a droit d'ôter à ses sujets la liberté du choix, et de ne leur laisser que le parti de l'obéissance. L'édit de Nantes ayant été vérifié, après bien des difficultés du parlement, du clergé et de l'université, Henri IV dit aux évêques : «Vous m'avez exhorté de mon devoir ; je vous exhorte du vôtre. Faisons bien à l'envi les uns des autres. Mes prédécesseurs vous ont donné de belles paroles ; mais moi, avec ma jaquette, je vous donnerai de bons effets : je verrai vos cahiers, et j'y répondrai le plus favorablement qu'il me sera possible. » Et il répondit au parlement qui était venu lui faire des remontrances

«Vous me voyez en mon cabinet où je viens vous parler, non pas en habit royal, ni avec l'épée et la cape, comme mes prédécesseurs, mais vêtu comme un père de famille, en pourpoint, pour parler familièrement à ses enfants. Ce que j'ai à vous dire est que je vous prie de vérifier l'édit que j'ai accordé à ceux de la religion. Ce que j'en ai fait est pour le bien de la paix. Je l'ai faite au-dehors ; je veux la faire au-dedans de mon royaume. » Après leur avoir 
exposé les raisons qu'il avait eues de faire l'édit, il ajouta : «Ceux qui empêchent que mon édit ne passe veulent la guerre ; je la déclarerai demain à ceux de la religion ; mais je ne la ferai pas ; je les y enverrai. J'ai fait l'édit ; je veux qu'il s'observe. Ma volonté devrait servir de raison; on ne la demande jamais au prince dans un État obéissant. Je suis roi. Je vous parle en roi. Je veux être obéi. »

Voilà comment il convient à un monarque de parler à ses sujets, quand il a évidemment la justice de son côté ; et pourquoi ne pourrait-il pas ce que peut tout homme qui a l'équité de son côté ? Quant aux sujets, la première loi que la religion, la raison et la nature leur imposent, est de respecter eux-mêmes les conditions du contrat qu'ils ont fait, de ne jamais perdre de vue la nature de leur gouvernement ; en France, de ne point oublier que tant que la famille régnante subsistera par les mâles, rien ne les dispensera jamais de l'obéissance ; d'honorer et de craindre leur maître, comme celui par lequel ils ont voulu que l'image de Dieu leur fût présente et visible sur la terre ; d'être encore attachés à ces sentiments par un motif de reconnaissance de la tranquillité et des biens dont ils jouissent à l'abri du nom royal ; si jamais il leur arrivait d'avoir un roi injuste, ambitieux et violent, de n'opposer au malheur qu' un seul remède, celui de l'apaiser par leur soumission, et de fléchir Dieu par leurs prières ; parce que ce remède est le seul qui soit légitime, en conséquence du contrat de soumission juré au prince régnant anciennement, et à ses descendants par les mâles, quels qu'ils puissent être ; et de considérer que tous ces motifs qu'on croit avoir de résister, ne sont, à les bien examiner, qu'autant de prétextes d'infidélités subtilement colorées ; qu'avec cette conduite, on n'a jamais corrigé les princes ni aboli les impôts ; et qu'on a seulement ajouté aux malheurs dont on se plaignait déjà, un nouveau degré de misère. Voilà les fondements sur lesquels les peuples et ceux qui les gouvernent pourraient établir leur bonheur réciproque.

Fin de l'article. 